\title{
Long Noncoding RNA CRNDE Promotes Proliferation of Gastric Cancer Cells by Targeting miR-145
}

\author{
Cheng En Hua Pei Zhun Duª Hui Dong Zhang ${ }^{b}$ Guang Jian Huanga \\ aDepartment of General Surgery, Huashan Hospital, Fudan University, bDepartment of General Surgery, \\ Shanghai Children's Medical Center, Shanghai, China
}

\author{
Key Words \\ LncRNA • CRNDE • miR-145 • E2F3 • Proliferation • Gastric cancer
}

\begin{abstract}
Background/Aims: The colorectal neoplasia differentially expressed (CRNDE) gene is a long noncoding RNA (IncRNAs) that is upregulated in colorectal cancer and glioma. Here, we investigated the regulatory function of CRNDE in gastric cancer (GC). Methods: CRNDE and miR-145 expression were assayed by qRT-PCR, and E2F3 protein expression was measured by western blotting. A luciferase reporter assay was used to detect the direct regulation of miR-145 by CRNDE. Cell viability and colony formation of human GC cells were detected using MTT and colony formation assay, respectively. Results: CRNDE was highly expressed in GC cell lines and tissues; overexpression of CRNDE increased GC cell viability and promoted colony formation. Knockdown of CRNDE did not result in loss of expression-related effects on cell proliferation and colony formation. Further investigation revealed that the miR-145 target gene E2F3 was strongly expressed following CRNDE competitive molecular sponging of miR145. Conclusion: CRNDE acted as a growth-promoting InCRNA in GC and maybe a potential target of GC treatment.

\section{Introduction}

Gastric cancer (GC) is the second most common cause of death from cancer worldwide [1]. Despite advances in therapy, the overall survival rate of patients with advanced GC is low. Long noncoding RNAs (lncRNAs) are transcripts that are longer than 200 nucleotides and have no protein coding function [2]. Some lncRNAs are known to be involved in complex mechanisms underlying the development of malignancies, including carcinogenesis, progression, and metastasis [3, 4], but the underlying molecular events are unknown.

Many lncRNAs regulate gene expression at several levels including transcription and post-transcriptional processing [5]. In a recently described regulatory mechanism, lncRNAs behave as competing endogenous RNAs (ceRNAs), acting as molecular sponges of microRNAs 
(miRNAs) to derepress miRNA targets [6]. For example, the lncRNA MALAT1 functions as a ceRNA to regulate cell division control (Cdc) 42 expression by sponging the miR-1 in human breast cancer [7]. H19 contributes to gallbladder cancer cell proliferation by modulating miR-194-5p targeting of AKT2 [8]. LncRNA UCA1, a ceRNA of miR-193a-3p, is active in nonsmall cell lung cancer carcinogenesis, and may be a potential target of antineoplastic therapy [9]. These findings suggest the involvement of lncRNAs in GC tumorigenesis.

Colorectal neoplasia differentially expressed (gene symbol CRNDE) is a non-proteincoding human gene locus that is upregulated in colorectal adenomas and carcinomas [10]. CRNDE transcripts are lncRNAs, a class of noncoding RNA having more than 200 base pairs. The lncRNA CRNDE is thought to be involved in tumorigenesis because its expression is increased in several cancers including colorectal and ovarian cancer, glioma, and hepatocellular carcinoma [11]. However, CRNDE activity has not been previously reported in GC.

In this study, we found that upregulation of CRNDE in GC cells and tissues were associated with increased GC cell viability and promotion of colony formation. Our analysis indicated that CRNDE functioned as a ceRNA to regulate the expression of E2F transcription factor 3 (E2F3) by competing for miR-145 binding and thereby promoting GC growth. This study adds to our understanding of GC pathogenesis.

\section{Materials and Methods}

\section{Tissue collection}

Twenty pairs of GC tissues and adjacent nonmalignant gastric tissue samples were obtained from Huashan Hospital (Shanghai, China). The study was approved by the Research Ethics Committee of the Medical Ethics Committee of Huashan Hospital. Informed consent was obtained from all patients. Specimens were immediately snap-frozen in liquid nitrogen and stored at $-80{ }^{\circ} \mathrm{C}$ until processing.

\section{Cell culture}

The SGC7901, BGC823, MGC803, and AGS human gastric cancer cell lines and GES-1 normal gastric epithelial cell line were obtained from the Chinese Institute of Biochemistry and Cell Biology (Shanghai, China). Cells were cultured in RPMI 1640 Medium (Invitrogen, Carlsbad, CA, USA) containing 10\% fetal bovine serum, $100 \mathrm{U} / \mathrm{ml}$ penicillin, and $100 \mathrm{U} / \mathrm{ml}$ streptomycin in culture flasks at $37^{\circ} \mathrm{C}$ with $5 \% \mathrm{CO}_{2}$.

\section{Quantitative real-time PCR ( $q R T$-PCR)}

Total RNA was extracted from cells and tissue samples using TRIzol reagent (Invitrogen, California, USA), following the manufacturer's instructions. Complementary DNA synthesis was performed using Prime Script reverse transcriptase reagent kit (TaKaRa, Dalian, China) according to the manufacturer's instructions; RNA was reverse transcribed to cDNA with a reverse transcription kit (TaKaRa, Dalian, China); and CRNDE, miR-145; and E2F3 expression were assayed by qRT-PCR using SYBR Premix Ex Taq (TaKaRa, Dalian, China). The PCR primer sequences used are listed as the following: CRNDE: $5^{\prime}$-GTAGGATGCCACTG-

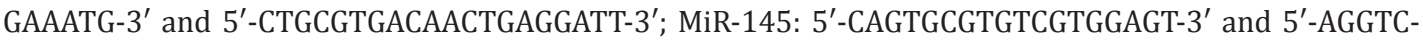
CAGTTTTCCCAGG-3'; E2F3: 5'-ATATCCCTAAACCCGCTTCC-3' and 5'-TGGTCCTCAGTCTGTAAGA-3'; mRNA, lncRNA, and miRNA expression were normalized against human GAPDH and U6 small nuclear (sn)RNA. Fold-change in expression was calculated by the relative quantification $\left(2^{-\Delta \Delta C t}\right)$ method.

\section{Lentivirus infection and establishment of stable cell lines}

Lentivirus expressing CRNDE, short hairpin (sh) RNA targeting CRNDE and their corresponding controls were purchased from GenePharma (Shanghai, China), named LV-CRNDE and LV-shCRNDE. The sequence of shCRNDE and negative control (NS) were shown: shCRNDE: $5^{\prime}$-CACCGGAAGGAGGAGATTCTGAAGATTCAAGAGATCTTCAGAATCTCC TCCTTCCTTTTTG-3', NS: 5'-CACCGTTCTCCGAACGTGTCACGTCAAGAGATTACGTG ACACGTTCGGAGAATTTTTTG-3'[12]. SGC-7901, BGC-823 and GES-1 cells were transduced with the recombinant lentivirus, and stable cell lines were established. miR-145 mimics were also obtained from GenePharma.

\section{KARGER}




\section{Cellular Physiology Cell Physiol Biochem 2017;42:13-21

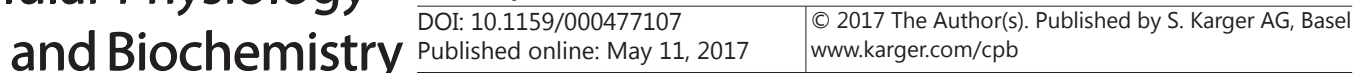

Hu et al.: LncRNA CRNDE Promotes GC Cell Proliferation

\section{Western blotting}

Western blotting was performed as previously described [13]. Total protein was extracted from cells using RIPA lysis buffer (Beyotime, Jiangsu, China). Samples of cell lysate protein were separated by sodium dodecyl sulfate polyacrylamide gel electrophoresis (SDS-PAGE) and transferred onto polyvinylidene fluoride (PVDF) membranes. After blocking with 5\% non-fat milk in TBS-T, proteins were then labelled with anti-E2F3 and anti-GAPDH (Cell Signaling Technology, Beverly, MA, USA) primary and secondary antibodies and detected with Image Acquisition using Image Quant ${ }^{\mathrm{TM}}$ LAS 4000 (GE Healthcare Life Sciences, Michigan, USA).

Cell proliferation assay

Cell proliferation was measured by 3-(4, 5-dimethylthiazole-2-yl)-2, 5-diphenyltetrazolium bromide (MTT) assay and colony formation. For the MTT assay, LV-infected cells were seeded into 96-well plates at a density of 2000 cells/well and cultured for 24,48 , or 72 hours. The spectrophotometric absorbance of each well was measured at $490 \mathrm{~nm}$ at different time points using a microplate reader absorbance test plate (Molecular Devices, Sunnyvale, CA, USA). The colony formation assay was assayed as previously described [14]. Briefly, infected cells were seeded into six-well plates at a density of 500 cells per well and cultured at $37{ }^{\circ} \mathrm{C}$ with $5 \% \mathrm{CO}_{2}$ humidified air for 2 weeks. Colonies were fixed and stained with $0.1 \%$ crystal violet $(1 \mathrm{mg} / \mathrm{ml})$, and the number of colonies was counted by light microscopy. The experiment was performed in triplicate and repeated 3 times. Plate efficiency $=$ (colony numbers / inoculated cell numbers) $\times 100 \%$.

Luciferase reporter assay

CRNDE fragments containing the putative binding sequence of miR-145 and its mutant sequence was cloned into a pGL3-control vector (Promega, Madison, WI, USA). The resulting vectors were sequenced and named CRNDE-WT, CRNDE-Mut. SGC7901 and BGC823 cells were cotransfected with the appropriate reporter plasmid, miRNA or pRL-TK Renilla plasmid (Promega) using Lipofectamine 2000 (Invitrogen). Luciferase activity was measured $48 \mathrm{~h}$ post-transfection using a dual-luciferase reporter assay system (Promega) following the manufacturer's instructions.

\section{RNA immunoprecipitation (RIP)}

To determine whether CRNDE was associated with the RNA-induced silencing complex (RISC), RNA immunoprecipitation (RIP) was performed using an EZ-Magna RIPRNA-binding protein immunoprecipitation kit (Millipore, Billerica, MA, USA) following the manufacturer's instructions. SGC7901 and BGC823 cell lysates containing CRNDE and miRNAs were prepared and incubated with RIP buffer containing magnetic beads conjugated to human anti-argonaute2 (Ago2) antibody (Millipore). Normal mouse IgG (Millipore) was used as a negative control. CRNDE and miRNAs present in the precipitates were assayed by qRT-PCR.

\section{Statistical analysis}

Data were reported as means \pm standard deviation (SD) and analyzed using SPSS 17.0 software (SPSS Inc., Chicago, IL, USA). Between-group differences were tested for significance using Student's $t$-test and one-way analysis of variance. Pearson correlation coefficients were calculated to determine the significance of the relationship of CRNDE and miR-145 expression. $P$-values $<0.05$ were considered significant.

\section{Results}

CRNDE expression is increased in human GC tissue and cell lines

The level of lncRNA CRNDE expression was first examined in GC tissues and matched adjacent normal tissue samples. As shown in Fig. 1A, CRNDE expression was significantly higher in GC tissues than in normal tissue. When CRNDE expression in SGC-7901, BGC823, MGC-803, AGS and GES-1 cell lines was assayed by qRT-PCR, CRNDE expression was found to be significantly higher in GC cells than in normal GES-1 gastric epithelial cells (Fig. 1B). Collectively, the results showed that CRNDE was upregulated in GC. 
Fig. 1. CRNDE was highly expressed in GC tissues and cell lines. (A) Expression of CRNDE was measured by qRT-PCR in 20 pairs of GC/ nontumor tissue specimens. (B) CRNDE expression was assayed in GC cell lines and normal gastric epithelial immortalized cells by qRTPCR. ${ }^{*} P<0.05$.
$A$

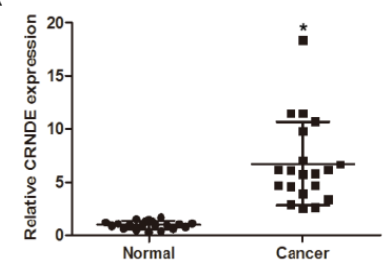

B

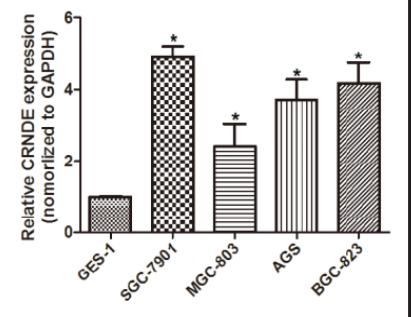

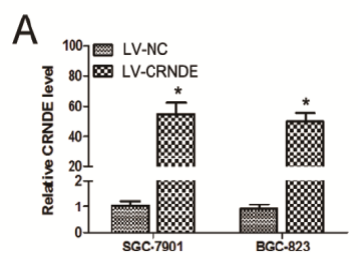
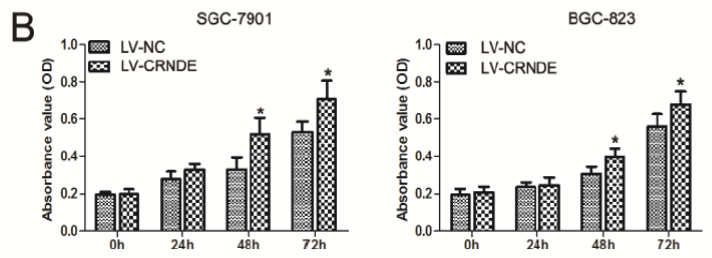

C
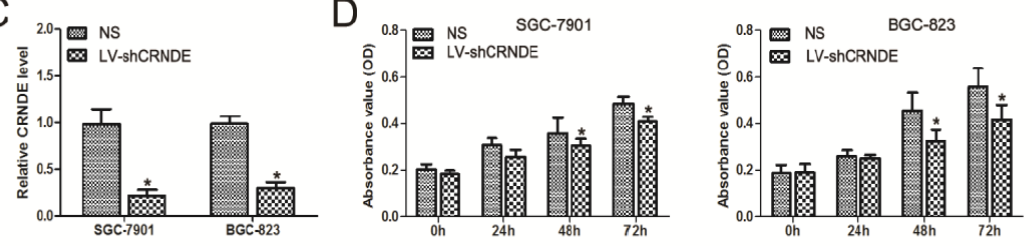

$E$
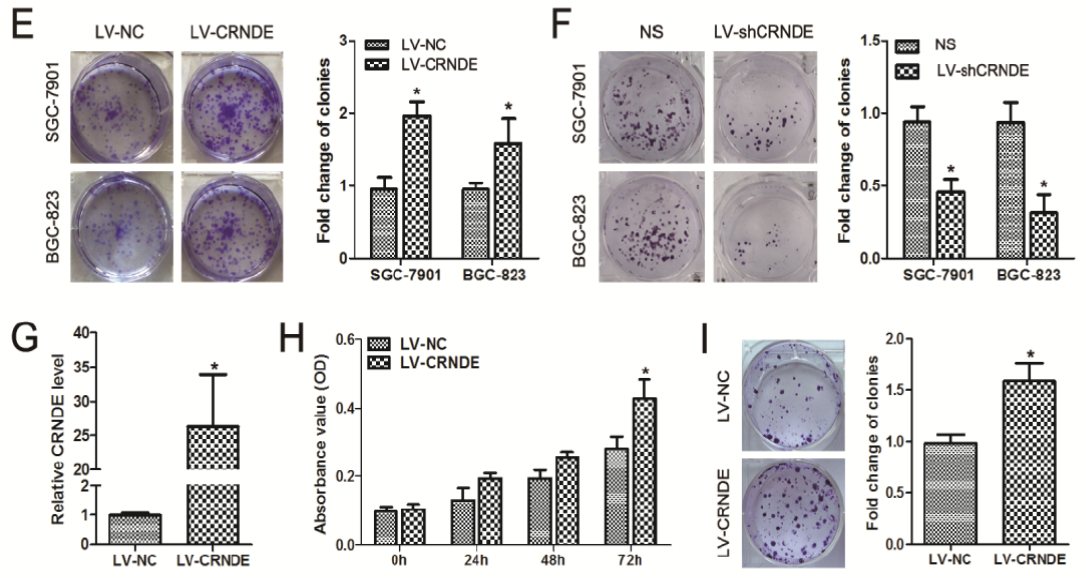

Fig. 2. CRNDE promoted proliferation of SGC-7901 and BGC-823 cells. (A) CRNDE expression was detected by qRT-PCR analysis in SGC-7901 and BGC-823 cells transfected with LV-CRNDE or LV-NC. (B) Cell viability was assayed by MTT assay. (C) CRNDE expression was measured in SGC-7901 and BGC-823 cell lines transfected with LV-shCRNDE or NS. (D) Cell viability was detected by MTT assay. (E) Representative images of colonies of LV-CRNDE- transfected SGC-7901 and BGC-823 cells. (F) Representative images of colonies of LV-shCRNDE-transfected SGC-7901 and BGC-823 cells. (G) CRNDE expression was detected by qRT-PCR analysis in GES-1 cells infected with LV-CRNDE or LV-NC. (H) Cell viability was assayed by MTT assay. (I) Representative images of colonies of LV-CRNDE-infected GES- 1 cells. ${ }^{*} P<0.05$.

\section{CRNDE promoted cell proliferation in GC}

To determine whether CRNDE regulated GC cell proliferation, we performed an in vitro gain and loss of function analyses of overexpression and silencing of CRNDE in SGC-7901 and BGC-823 gastric cancer cells (Fig. 2A and C). The MTT assay showed that overexpression of CRNDE significantly promoted proliferation of GC cells compared with normal controls (Fig. 2B). Proliferation of GC cells was significantly inhibited when CRNDE was silenced (Fig. 2D). Similarly, increased CRNDE expression was associated with formation of significantly 
A

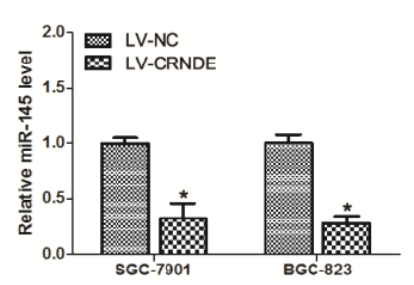

C

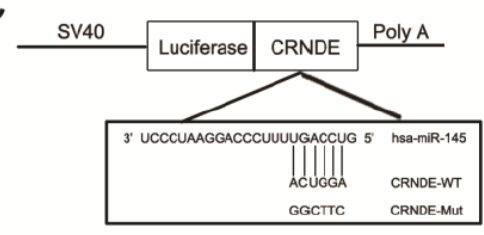

$E$

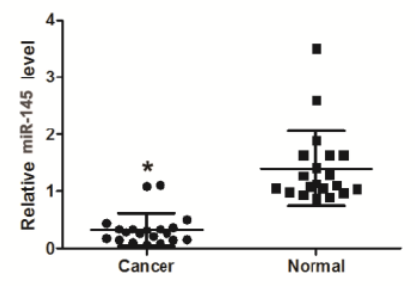

B

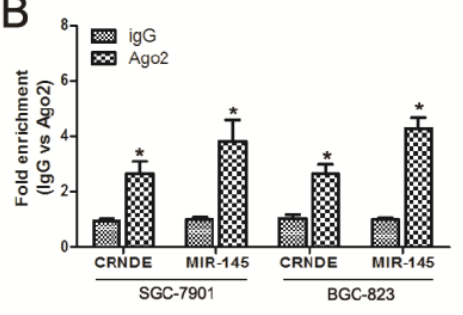

D

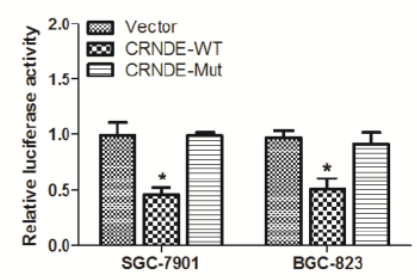

$\mathrm{F}$

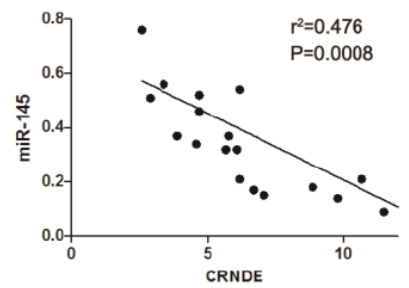

Fig. 3. CRNDE acted as a molecular sponge of miR-145. (A) miR-145 expression was examined in LV-CRNDE treated BGC-823 andSCG-7901 cells by qRT-PCR. (B) The association of CRNDE, miR-145 and Ago 2 was confirmed by assay of BGC-823 andSCG-7901 cells lysates by RNA immunoprecipitation with an Ago2 antibody. (C) Putative miR-145-binding sequence of CRNDE RNA. (D) Relative luciferase activities were measured in BGC-823 and SCG-7901 cells transfected with vector, CRNDE-WT, or CRNDE-Mut. ${ }^{*} P<0.05$ vs. vectors. (E) miR-145 expression was assayed in 20 pairs of gastric cancer tissues/non tumor tissue specimens. (F) Pearson correlation coefficients were calculated to determine the significance of the relation of CRNDE and miR-145 expression. ${ }^{*} P<0.05$.

more colonies of GC cells than in negative controls (Fig. 2E). Significantly fewer colonies formed in cultures of LV-shCRNDE GC cells than were seen in the negative control cells (Fig. $2 \mathrm{~F}$ ). Furthermore, GES-1 cells which have relatively lower endogenous CRNDE were infected with LV-CRNDE or LV-NC (Fig. 2G). Consistent with the above observations, up-regulating the expression of CRNDE results in increased viability and colony-formation ability of GC cells (Fig. 2H and I). These results confirmed the oncogenic role of CRNDE in GC.

\section{CRNDE acts as a molecular sponge of miR-145}

The ceRNA hypothesis presumes that specific lncRNA can act as sinks for pools of active miRNAs, functionally liberating mRNA transcripts targeted by that set of miRNAs. To determine whether CRNDE acted as a ceRNA, we used miRcode-target (http://www. mircode.org/) to predict potential lncRNA-miRNA interactions. We found that miR-145 was significantly decreased in SGC-7901 and BGC-823 cells treated with LV-CRNDE, and was among several miRNAs that had high predicted CRNDE binding high scores (Fig. 3A).

miRNAs have previously been shown to be present as miRNA ribonucleoprotein complexes that contain Ago2, the key RISC component $[15,16]$. To test whether CRNDE associated with a RISC complex, we examined miR-145 and CRNDE on magnetic beads conjugated to anti-Ago2 antibody. We found that miR-145 was highly enriched in GC cells (Fig. 3B), suggesting that CRNDE may act by deregulating miR-145. In addition, the activity of luciferase reporters containing the putative binding sequence of CRNDE in miR-145 was significantly decreased in CRNDE-WT constructs compared with CRNDE-Mut constructs, 
Hu et al.: LncRNA CRNDE Promotes GC Cell Proliferation

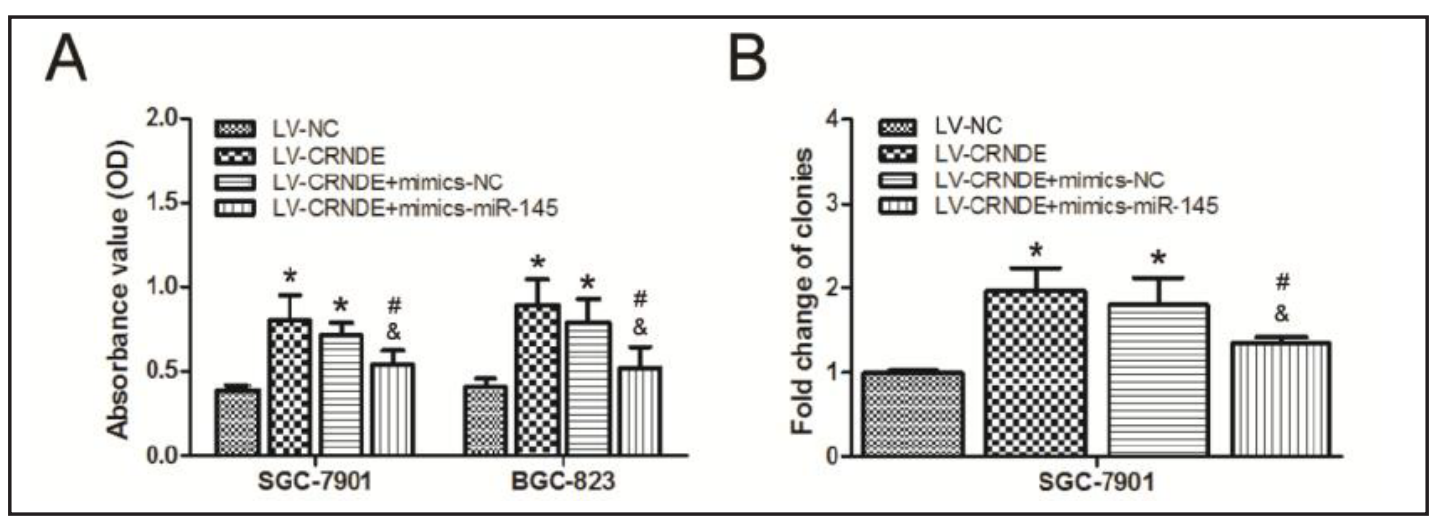

Fig. 4. CRNDE promoted GC cell proliferation and colony formation by competitively binding miR-145. (A) Cell proliferation was examined by MTT assay, (B) Colony formation assay in BGC-823 and SCG-791 cells. ${ }^{*} P<0.05$ vs. LV-NC group. ${ }^{\#} P<0.05$ vs. LV-CRNDE+mimics-NC group. ${ }^{\circledR} P<0.05$ vs. LV-CRNDE group.

which were not affected (Fig. 3C and D). Furthermore, miR-145 expression was significantly decreased in GC tissues compared with that in adjacent normal tissue samples (Fig. 3E), and miR-145 level had a significant negative correlation with CRNDE level (Fig.3F). Collectively, the results suggest that miR-145 was targeted by CRNDE.

miR-145 reverses the promoting effects of CRNDE on GC cells

To investigate whether the effects of miR-145 on cell proliferation were mediated by CRNDE, we transfected SGC-7901 and BGC-823 cells with miR-145 mimics and a CRNDE expression vector. The MTT assays showed that miR-145 abrogated the promotion of cell proliferation (Fig. 4A) and cell culture showed that colony formation was also inhibited (Fig. 4B). These observations suggest that CRNDE promoted tumor cell growth in part by competitively binding miR-145.

CRNDE modulated expression of endogenous miR-145 targets E2F3

miR-145 has been reported to target and repress E2F3 expression in human GC [17]. To confirm whether CRNDE promoted GC progression by targeting miR-145, we evaluated the effect of CRNDE on E2F3. We found that E2F3 protein, but not mRNA, expression was significantly enhanced in SGC-7901 and BGC-823 cells transfected with LV-CRNDE-WT, whereas LV-CRNDE-Mut had no significant effect on E2F3 expression (Fig. 5A and B). When we transfected SGC-7901 and BGC-823 cells with LV-shCRNDE, E2F3 protein, but not mRNA expression, was significantly reduced following CRNDE downregulation (Fig. 5C and D). SGC-7901 and BGC-823 cells were transfected with the vector, LV-CRNDE, or LV-CRNDE combined with miR-145 mimics to investigate the effect of miR-145 repression on E2F3 expression. E2F3 protein, but not mRNA, expression was increased by CRNDE, and that effect was partially repressed by miR-145 mimics (Fig. 5E and F). These results show that CRNDE abrogated the repression of E2F3 induced by miR-145 by sequestering endogenous miR-145, and exerted oncogenic functions by modulating miR-145/E2F3.

\section{Discussion}

Recent evidence of the role of noncoding RNAs in cancer pathogenesis has added to our understanding of the biology of this disease [7, 18-20], and in the past decade, study of miRNAs has dominated the field of noncoding RNA regulation. Recently, accumulated evidence on IncRNA has indicated that dysregulation of IncRNA may not only affect the regulation of the eukaryotic genome, but also provide a growth advantage to malignant cells, resulting in progressive and uncontrolled tumor growth [21, 22]. Therefore, lncRNAs may 


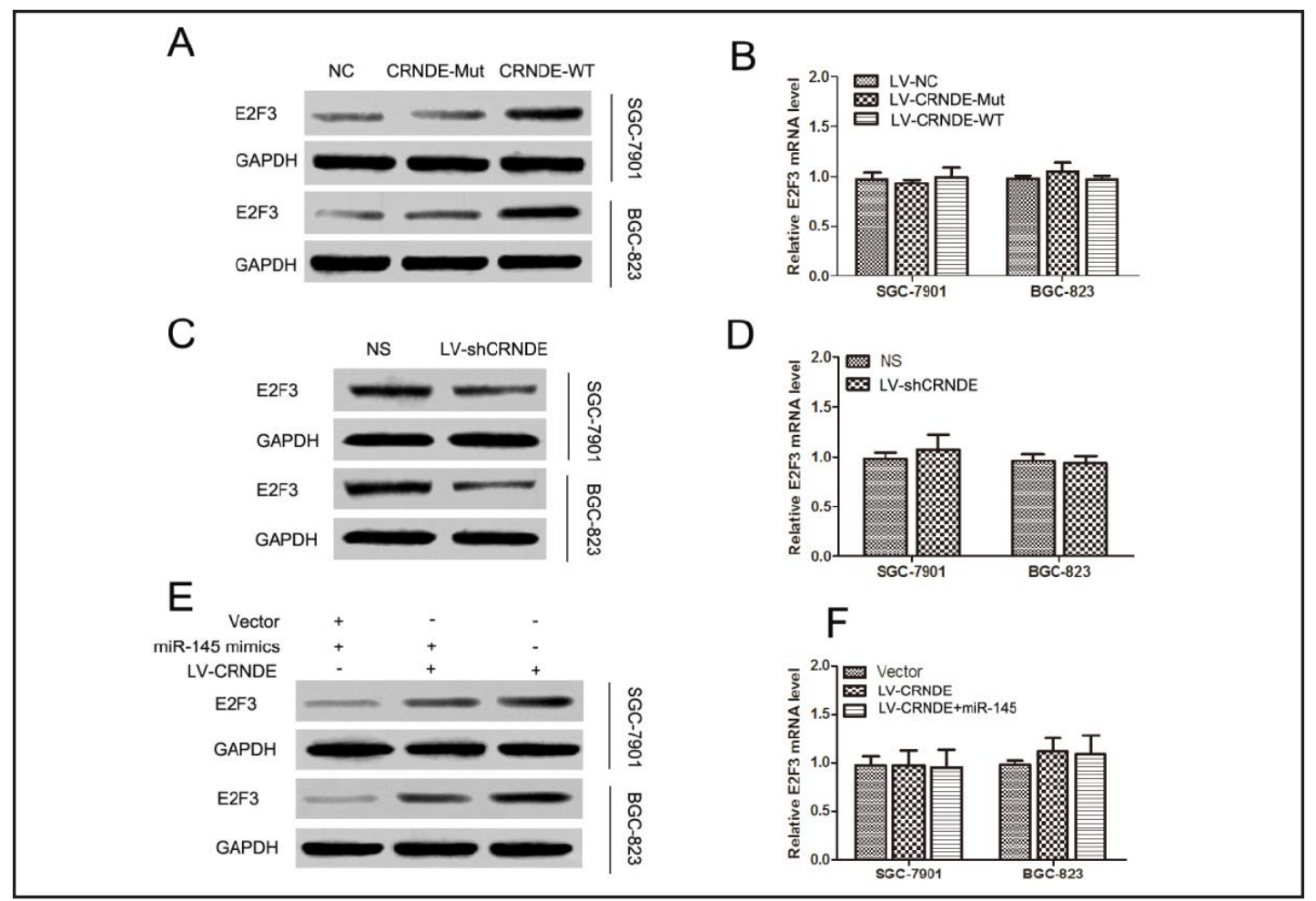

Fig. 5. CRNDE modulated the expression of the endogenous miR-145 target E2F3. (A, B) E2F3 protein and mRNA expression in BGC-823 and SCG-791 cells transfected with vector, LV-CRNDE-Mut, or LV-CRNDE-WT were measured by western blotting and qRT-PCR. (C, D) Expression of E2F3 protein and mRNA in BGC-823 and SCG-791 cells transfected with LV-shCRNDE or NS was measured by western blotting and qRT-PCR. (E, F) E2F3 protein and mRNA expression in BGC-823 and SCG-791 cells transfected with vector, LV-CRNDE, or LV-CRNDE combined with miR-145 mimics were assayed by western blotting and qRT-PCR.

provide a missing piece to help complete the puzzle of the oncogenic and tumor suppressor network.

CRNDE is located on chromosome 16, and was initially identified as an IncRNA in colorectal cancer. Its expression is significantly upregulated in colorectal cancer, where it is involved in cell proliferation, migration, and invasion [10]. A transcript isoform (CRNDE-h) found in patient plasma may serve as a biomarker [23]. The CRNDE IncRNA is also strongly upregulated in glioma, and contributes to disease progression by promoting cell proliferation, migration and invasion by mTOR signaling [24]. CRNDE is also overexpressed in melanoma and lymphocytic leukemia cells.

The activity of CRNDE in GC has not been previously described. In this study, we found that CRNDE was upregulated in GC cells and tissues, and overexpression significantly promoted cell proliferation and colony formation, whereas knockdown of CRNDE negatively regulated cell growth. The findings show that CRNDE played an important role in the modulation of GC progression, and warrant further research on CRNDE as a therapeutic target in GC.

Increasing numbers of reports reveal the existence of a widespread interaction network involving ceRNAs, where ncRNAs could regulate modulatory RNA by binding and titrating them off their binding sites on protein coding messengers $[6,25]$. This type of regulation has been shown by H19, NEAT1, ROR and PCGEM1 [26-29]. We hypothesized that IncRNA CRNDE functioned as a ceRNA to promote GC progression, and looked for potential interactions with miRNAs. We used bioinformatics analysis and luciferase assays to verify the direct binding of predicted miRNA response elements on the CRNDE transcript. The results showed that miR145 could form complementary base pairing with CRNDE and could reduce expression of a pGL3-CRNDE reporter gene. In the RIP assay, expression of CRNDE immunoprecipitated with 
Ago2 was higher than that immunoprecipitated with IgG, indicating a reciprocal repression of CRNDE and miR-145 caused by RISC. We also showed that miR-145 expression level was negatively correlated with CRNDE level in GC tissue and that miR-145 overexpression arrested GC cell growth. The findings demonstrate that CRNDE interacted with miR-145 in GC pathogenesis.

miR-145 in known to inhibit tumor progression and metastasis by downregulating E2F3 in human GC [17], and to show the miRNA-related activity of CRNDE in GC pathogenesis, we evaluated its effect on E2F3, which is a target of miR-145.The E2F family of transcription factors regulates both cellular proliferation and differentiation, and E2F3 is a key transcriptional factor of genes that control the proliferation rate of both primary and tumor cells [30]. A number of studies have reported that E2F3 is targeted by various miRNAs and is involved in the genesis and progression of human cancers [31-34]. Our study showed that E2F3 expression was significantly increased by upregulating CRNDE expression, whereas shCRNDE significantly decreased E2F3 expression. The results indicate that CRNDE oncogenesis involved functioning as a ceRNA to regulate E2F3 expression by sponging miR145 in GC.

In summary, we showed that the IncRNA CRNDE promoted GC cell proliferation by competitively binding miR-145, and described a novel CRNDE/miR-145/E2F3 signaling pathway with a regulatory function in GC. The findings show that CRNDE may be a target for GC therapy, with the crosstalk between miR-145, CRNDE and E2F3 shedding new light on potential treatment of GC.

\section{Disclosure Statement}

The authors declare there are no conflicts of interest.

\section{References}

1 Jemal A, Bray F, Center MM, Ferlay J, Ward E, Forman D: Global cancer statistics. CA Cancer J Clin 2011;61:69-90.

2 Ponting CP, Oliver PL, Reik W: Evolution and functions of long noncoding rnas. Cell 2009;136:629-641.

-3 Hung CL, Wang LY, Yu YL, Chen HW, Srivastava S, Petrovics G, Kung HJ: A long noncoding rna connects c-myc to tumor metabolism. Proc Natl Acad Sci U S A 2014;111:18697-18702.

4 Haemmerle M, Gutschner T: Long non-coding rnas in cancer and development: Where do we go from here? Int J Mol Sci 2015;16:1395-1405.

-5 Mercer TR, Dinger ME, Mattick JS: Long non-coding rnas: Insights into functions. Nat Rev Genet 2009;10:155-159.

6 Salmena L, Poliseno L, Tay Y, Kats L, Pandolfi PP: A cerna hypothesis: The rosetta stone of a hidden rna language? Cell 2011;146:353-358.

7 Chou J, Wang B, Zheng T, Li X, Zheng L, Hu J, Zhang Y, Xing Y, Xi T: Malat1 induced migration and invasion of human breast cancer cells by competitively binding mir-1 with cdc42. Biochem Biophys Res Commun 2016;472:262-269.

8 Wang SH, Wu XC, Zhang MD, Weng MZ, Zhou D, Quan ZW: Long noncoding rna h19 contributes to gallbladder cancer cell proliferation by modulated mir-194-5p targeting akt2. Tumour Biol 2016;37:97219730.

-9 Nie W, Ge HJ, Yang XQ Sun X, Huang H, Tao X, Chen WS, Li B: Lncrna-uca1 exerts oncogenic functions in non-small cell lung cancer by targeting mir-193a-3p. Cancer Lett 2016;371:99-106.

-10 Graham LD, Pedersen SK, Brown GS, Ho T, Kassir Z, Moynihan AT, Vizgoft EK, Dunne R, Pimlott L, Young GP, Lapointe LC, Molloy PL: Colorectal neoplasia differentially expressed (crnde), a novel gene with elevated expression in colorectal adenomas and adenocarcinomas. Genes Cancer 2011;2:829-840.

-11 Esposti DD, Hernandez-Vargas H, Voegele C, Fernandez-Jimenez N, Forey N, Bancel B, Le Calvez-Kelm F, McKay J, Merle P, Herceg Z: Identification of novel long non-coding rnas deregulated in hepatocellular carcinoma using rna-sequencing. Oncotarget 2016;7:31862-31877. 


\section{Cellular Physiology Cell Physiol Biochem 2017;42:13-21

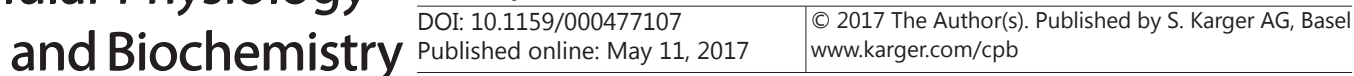

Hu et al.: LncRNA CRNDE Promotes GC Cell Proliferation

12 Zheng J, Li XD, Wang P, Liu XB, Xue YX, Hu Y, Li Z, Li ZQ, Wang ZH, Liu YH: Crnde affects the malignant biological characteristics of human glioma stem cells by negatively regulating mir-186. Oncotarget 2015;6:25339-25355.

13 Zhao X, Wang P, Liu J, Zheng J, Liu Y, Chen J, Xue Y: Gas5 exerts tumor-suppressive functions in human glioma cells by targeting mir-222. Mol Ther 2015;23:1899-1911.

14 Chen DL, Zeng ZL, Yang J, Ren C, Wang DS, Wu WJ, Xu RH: L1cam promotes tumor progression and metastasis and is an independent unfavorable prognostic factor in gastric cancer. J Hematol Oncol 2013;6:43.

-15 Izaurralde E: Elucidating the temporal order of silencing. EMBO Rep 2012;13:662-663.

-16 Filipowicz W, Bhattacharyya SN, Sonenberg N: Mechanisms of post-transcriptional regulation by micrornas: Are the answers in sight? Nat Rev Genet 2008;9:102-114.

17 Chang S, Gao L, Yang Y, Tong D, Guo B, Liu L, Li Z, Song T, Huang C: Mir-145 mediates the antiproliferative and gene regulatory effects of vitamin $\mathrm{d} 3$ by directly targeting e2f3 in gastric cancer cells. Oncotarget 2015;6:7675-7685.

18 Li S, Zhang H, Ning T, Wang X, Liu R, Yang H, Han Y, Deng T, Zhou L, Zhang L, Bai M, Wang X, Ge S, Ying G, Ba Y. MiR-520b/e Regulates Proliferation and Migration by Simultaneously Targeting EGFR in Gastric Cancer. Cell Physiol Biochem. 2016;40(6):1303-1315.

19 Zhu P, Zhang J, Zhu J, Shi J, Zhu Q, Gao Y. MiR-429 Induces Gastric Carcinoma Cell Apoptosis Through Bcl-2. Cell Physiol Biochem. 2015;37(4):1572-80.

20 Zhang Z, Zhu Z, Watabe K, Zhang X, Bai C, Xu M, Wu F, Mo YY: Negative regulation of lncrna gas5 by mir-21. Cell Death Differ 2013;20:1558-1568.

-21 Serviss JT, Johnsson P, Grander D: An emerging role for long non-coding rnas in cancer metastasis. Front Genet 2014;5:234.

22 Moran VA, Perera RJ, Khalil AM: Emerging functional and mechanistic paradigms of mammalian long noncoding rnas. Nucleic Acids Res 2012;40:6391-6400.

23 Ellis BC, Molloy PL, Graham LD: Crnde: A long non-coding rna involved in cancer, neurobiology, and development. Front Genet 2012;3:270.

24 Wang Y, Li J, Zhang Y, Yin H, Han B: Crnde, a long-noncoding rna, promotes glioma cell growth and invasion through mtor signaling. Cancer Lett 2015;367:122-128.

25 Jalali S, Bhartiya D, Lalwani MK, Sivasubbu S, Scaria V: Systematic transcriptome wide analysis of lncrnamirna interactions. PLoS One 2013;8:e53823.

26 Liu G, Xiang T, Wu QF, Wang WX: Long noncoding rna h19-derived mir-675 enhances proliferation and invasion via runx1 in gastric cancer cells. Oncol Res 2016;23:99-107.

27 Wang P, Wu T, Zhou H, Jin Q He G, Yu H, Xuan L, Wang X, Tian L, Sun Y, Liu M, Qu L: Long noncoding rna neat1 promotes laryngeal squamous cell cancer through regulating mir-107/cdk6 pathway. J Exp Clin Cancer Res 2016;35:22.

28 Gao S, Wang P, Hua Y, Xi H, Meng Z, Liu T, Chen Z, Liu L: Ror functions as a cerna to regulate nanog expression by sponging mir-145 and predicts poor prognosis in pancreatic cancer. Oncotarget 2016;7:1608-1618.

-29 Kang Y, Song J, Kim D, Ahn C, Park S, Chun CH, Jin EJ: Pcgem1 stimulates proliferation of osteoarthritic synoviocytes by acting as a sponge for mir-770. J Orthop Res 2016;34:412-418.

30 Humbert PO, Verona R, Trimarchi JM, Rogers C, Dandapani S, Lees JA: E2f3 is critical for normal cellular proliferation. Genes Dev 2000;14:690-703.

-31 Li X, Li H, Zhang R, Liu J: Microrna-449a inhibits proliferation and induces apoptosis by directly repressing e2f3 in gastric cancer. Cell Physiol Biochem 2015;35:2033-2042.

-32 Geng D, Song X, Ning F, Song Q Yin H: Mir-34a inhibits viability and invasion of human papillomaviruspositive cervical cancer cells by targeting e2f3 and regulating survivin. Int J Gynecol Cancer 2015;25:707713.

-33 Liu L, Qiu M, Tan G, Liang Z, Qin Y, Chen L, Chen H, Liu J: Mir-200c inhibits invasion, migration and proliferation of bladder cancer cells through down-regulation of bmi-1 and e2f3. J Transl Med 2014;12:305.

-34 Xue J, Niu YF, Huang J, Peng G, Wang LX, Yang YH, Li YQ: Mir-141 suppresses the growth and metastasis of hcc cells by targeting e2f3. Tumour Biol 2014;35:12103-12107. 